\title{
3-D Hand Pose Estimation from Kinect's Point Cloud Using Appearance Matching
}

\author{
Pasquale Coscia \\ Francesco A.N. Palmieri \\ Francesco Castaldo \\ Alberto Cavallo
}

Seconda Universitá di Napoli (SUN),

Dipartimento di Ingegneria Industriale e dell'Informazione, via Roma 29, 81030 Aversa (CE) - Italy

\author{
PASQUALE.COSCIA@UNINA2.IT \\ FRANCESCO.PALMIERI@UNINA2.IT \\ FRANCESCO.CASTALDO@UNINA2.IT \\ ALBERTO.CAVALLO@UNINA2.IT
}

\begin{abstract}
We present a novel appearance-based approach for pose estimation of a human hand using the point clouds provided by the low-cost Microsoft Kinect sensor. Both the free-hand case, in which the hand is isolated from the surrounding environment, and the hand-object case, in which the different types of interactions are classified, have been considered. The handobject case is clearly the most challenging task having to deal with multiple tracks. The approach proposed here belongs to the class of partial pose estimation where the estimated pose in a frame is used for the initialization of the next one. The pose estimation is obtained by applying a modified version of the Iterative Closest Point (ICP) algorithm to synthetic models to obtain the rigid transformation that aligns each model with respect to the input data. The proposed framework uses a "pure" point cloud as provided by the Kinect sensor without any other information such as RGB values or normal vector components. For this reason, the proposed method can also be applied to data obtained from other types of depth sensor, or RGB-D camera.
\end{abstract}

Keywords: Hand Pose, Kinect, Point Cloud, RANSAC, DBSCAN, ICP

\section{Introduction}

Hand pose estimation, by means of one or more cameras, is a desired feature of many information systems in several applications both in navigation and manipulation. For example in robotic applications, where robots are equipped with dexterous hands, it is important to provide feedback to the robot itself and/or to qualify how well it may be performing human-like grasps. In manipulators, efficient processing for analyzing hand motions may be an important part of a system specially when a device has to control complex machinery with many degrees of freedom (DoFs).

Currently, the best results for capturing hand motion are obtained by means of visual markers, electro-mechanical/magnetic sensing devices, or other specifically designed hardware. They provide a complete set of measurements for all hand's functionalities. Unfortunately, they have several disadvantages in term of casual use as they are invasive, expensive, restrict the naturalness of motion and require complex calibration and setup in order to obtain accurate measurements. Therefore, there is a great interest in developing 
markerless based solutions because of their potential to provide more natural interaction. Despite the significant progress in this field, the problem remains still open and reveals various theoretical and practical challenges due to a large number of issues. Fundamentally, the main problems encountered in the design of hand pose estimation systems include: high-dimensionality, self-occlusions, speed processing and hand speed.

As stated in Erol et al. [1], there are two main approaches to the hand pose estimation problem: partial and full DoF. Partial means that we do not have all the kinematics parameters of the hand's skeleton, but only rough information about the hand motion, such as position of the palm or the fingers. Contrariwise, full DoF pose estimation approaches attempt to estimate all the kinematic parameters of the hand skeleton.

The possibility to use depth information with a relatively low-cost sensors has given new perspectives to the hand tracking. Among all, the Kinect sensor [2] has become one of the most used depth sensor. The development of different versions of such device over the years, with increasingly high performance, proves its importance in the fields of gaming and research. It combines two technology, a RGB camera and an IR camera. To obtain depth information it uses the structured light principle. The idea behind this principle is to project a known pattern onto the scene and infer the depth of the objects using the deformation of such pattern and a triangulation method.

The Kinect has pioneered the development of new types of depth sensors such as the 3Gear Systems interface [3], the Leap Motion controller [4] or the most recent Creative Senz3D camera [5]. The 3Gear Systems interface is able to estimate hand poses using two Kinect cameras. The Leap Motion technology enables users to manipulate virtual objects with hand motions. The Creative Senz3D performs a close-range hand and finger tracking as well as facial analysis. However, more research effort is needed to understand the real potentialities of these devices.

The output of depth sensors is typically a point cloud that has become one of the most common framework for object representation in computer vision. A point cloud is a data structure containing a set of multi-dimensional points expressed within a given coordinate system and is typically used to represent the external surface of an object. In a threedimensional coordinate system, these points usually stand for the $x, y$ and $z$ geometric coordinates of the sampled surface. Each point may also have other attributes such as the components of the normal vector, the accuracy, or the color in the four spectral bands. In this work only the $x y z$-coordinates of the point clouds have been used.

The main contribution of this paper is a method, based on a combination of computer vision algorithms, for estimating human hand pose during different static gestures as well as predefined grasping tasks. Throughout the paper different methods and algorithms are carefully selected to achieve the best results in terms of reliability and robustness of the recognition task.

The rest of the paper is organized as follows: Section 2 provides a brief overview of the state of the art regarding the hand pose estimation problem. Section 3 presents in detail each step of the proposed framework and their relative outputs. Section 4 shows experimental results for a number of real test sequences. The last section discusses the results of the proposed framework and suggestions for improvements. 


\section{Related work}

The 3-D hand pose estimation problem has been addressed by many authors, including Stenger et al. [6], Rehg and Kanade [7], de La Gorce [8] for a single RGB camera and de Campos and Murray [9] for multiple cameras. Athitsos et al. [10] estimate 3-D hand pose from cluttered images. They formulate the hand pose estimation as an image indexing problem, using a large database of synthetic hand images. They also use an approximation of the image-to-model chamfer distance and a probabilistic line matching method to retrieve the closest matches for an input hand from the database. Rosales et al. [11] propose a system for recovering 3-D hand pose from monocular color sequences. Their system employs a nonlinear supervised learning framework where the training set is obtained via a CyberGlove. Several approaches have also been proposed to estimate the hand pose from depth images. Mo et al. [12] use a laser-based camera to produce low-resolution depth images. The method is based on recognizing hand poses as finger states. Malassiotis et al. [13] extract PCA features from depth images of synthetics 3-D hand models for training. Liu et Fujimura [14] recognize hand gestures by using a sequence of real-time depth image data acquired by an active sensing hardware. Their approach makes full use of 3-D trajectory as well as shape information for classifying gestures. Suryanarayan et al. [15] use depth information to recognize scale and rotation invariant hand poses dynamically. They have designed a volumetric shape descriptor enfolding the hand to generate a 3 -D cylindrical histogram and to achieve a real-time pose recognition.

A milestone is represented by the work of Oikonomidis et al. [16]. They propose a trackingbased method by making use of a RGB-D Kinect camera. They treat the pose estimation as an optimization problem, using a variant of Particle Swarm Optimization (PSO), selecting parameters that minimize the error between the 3-D hypothesized instances and visual observations of the hand. The method, however, requires an explicit initialization step for better results. They also assert that their algorithm can run at about 15 FPS if it is implemented on a GPU that corresponds to only half the rate at which the Kinect can operate. More recently, the approach proposed by Melax et al. [17] for 3-D markerless hand tracking from a depth camera uses an augmented rigid body simulation that allows to handle tracking as a linear complementary problem. To limit motion, the system generates constraints that are resolved with a projected Gauss-Seidel solver.

Different approaches have also been proposed to deal with the interaction with objects. In particular, Fu and Santello [18] have created a framework for tracking hand and object during grasping tasks. Grasping tasks are modeled as a collision detection problem while the hand is tracked with a marker-based solution using an Extended Kalman Filter. Hamer et al. [19] address the problem of manipulating objects using individual local tracker for each part of an articulated structure. A collection of surfaces patches forms the hand and a Markov random field enforces valid configurations connecting the segments.

\section{Our Approach}

Fig. 1 shows the block diagram of the proposed framework. The first step is background subtraction followed by clustering and matching steps in order to estimate the hand pose. Since the output of the matching step is fed back for the next frame, there is no real 


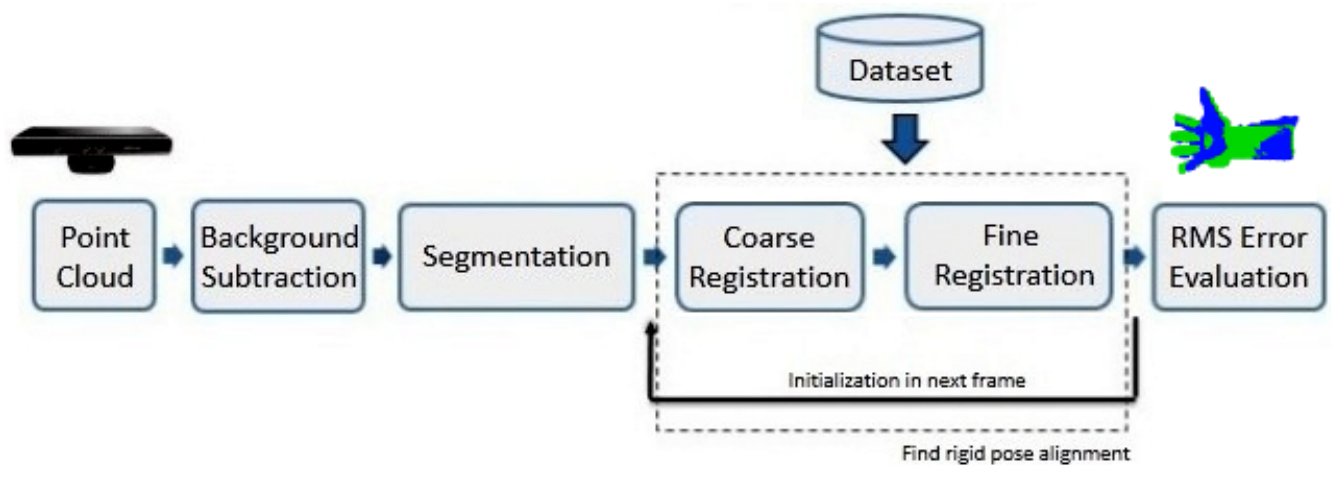

Figure 1: Overview of the proposed framework for 3-D hand pose estimation.

tracking. The starting point of the search, at each frame, is based on the result of the previous one. The pose is represented as a frame-dependent rotation matrix $\mathbf{R}[\mathrm{k}]$ and a translation vector $\mathbf{t}[\mathrm{k}]$ that update the position of the models' point clouds $\mathbf{M}_{i}$ in the space: $\mathbf{M}_{i}[k]=\mathbf{R}[k] \cdot \mathbf{M}_{i}[k-1]+\mathbf{t}[k]$.

Our case study consists of a scene in which the hand is located in front of the sensor and has a plane background. For grasping tasks, it has been considered only one object at time, situated on a table. The hand and the objects are placed at a fixed distance. Therefore there is no need to change the scale of the model. The point clouds have been stored at approximately $30 \mathrm{~Hz}$ and then processed. Each of them, before the processing, contains approximately 200k 3-D points. The point clouds have been captured using the Simulink Support for Kinect [20] and all the algorithms have been written in MATLAB.

\subsection{Background Subtraction}

The first step in processing the data consists in removing the background, that in our case was roughly a plane (a wall). To perform the subtraction, we used the RANSAC algorithm. RANSAC stands for "RANdom SAmple Consensus" and it is an iterative method for a robust parameters estimation of a mathematical model from a set of data containing outliers. In our case the model is represented by a plane, while outliers are the hand and the objects. It is a nondeterministic algorithm because it produces the correct result only with a given probability, which raises with the increase of the number of iterations. Its main applications include 3-D models fitting, stereo matching and linear regression. Several variations of the algorithm have been proposed using different cost functions or adaptive parameters for better performance [21;22]. In this work, we used the function written by Kovesi [23], that uses the RANSAC algorithm to robustly fit a plane to a set of $3 \mathrm{D}$ data points. The algorithm operates as follows:

- Randomly select a subset $\mathbf{S}$ of the original data $\mathbf{P}$.

- Estimate the model from the subset $\mathbf{S}$.

- Test all data points $p_{i} \in \mathbf{P}$ that fit the estimated model, i.e., they are within a distance threshold $\tau$ with respect to the model. Those points represent the consensus set. 
- Re-estimate the model using all points of the consensus set if the number of inliers is greater than a predefined threshold, otherwise select a new subset $\mathbf{S}$ of points and estimate a new model.

- After $\mathrm{N}$ trials, among all consensus set, the largest set is selected.

The algorithm is described in detail in [24]. For all experiments, we set the maximum number of iterations and the threshold $\tau$ to 1000 and 0.1 respectively. Fig. 2 shows the result of the RANSAC algorithm during a grasping task. The background is completely removed without affecting the remaining point cloud represented by the hand and the object. Fig. 3 shows the results of the algorithm using different values of $\tau$. A high threshold means that there is high sensitivity towards the outliers, therefore some points that belong to the hand are eliminated. A low threshold implies that points that deviate slightly from the plane model are considered as outliers. A proper trade-off considering the distance of the objects from the background must be adopted.

Background subtraction eliminates much of the data points in the cloud, hence it can be managed more easily and quickly for the next steps where the number of 3-D points typically drops by an order of magnitude.

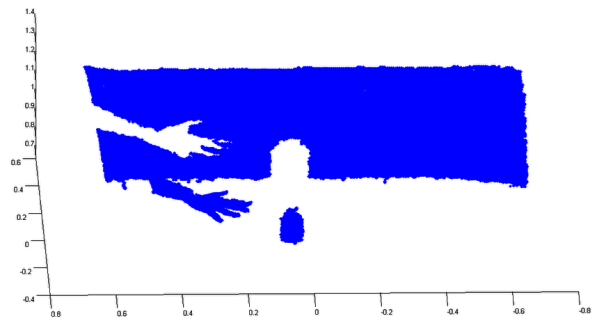

(a)

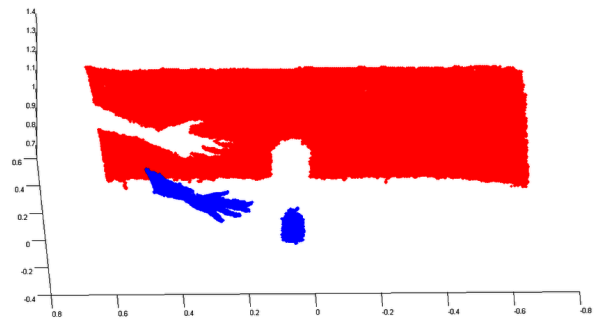

(b)

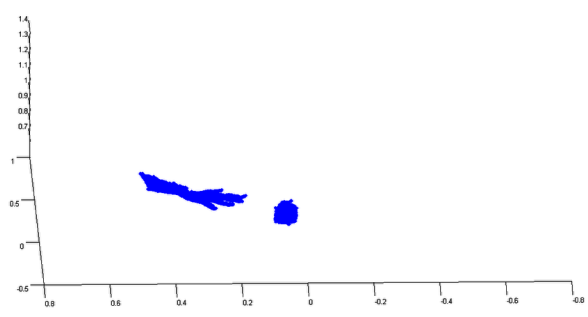

(c)

Figure 2: Results of applying RANSAC plane fitting algorithm: a) Original Point Cloud; b) Points (in red) that belong to the plane model determined by RANSAC; c) Point Cloud after background subtraction.

\subsection{Segmentation}

In order to associate each 3-D point to the corresponding cluster in the scene we have tested three different clustering algorithms: K-means, Euclidean Cluster Extraction and 


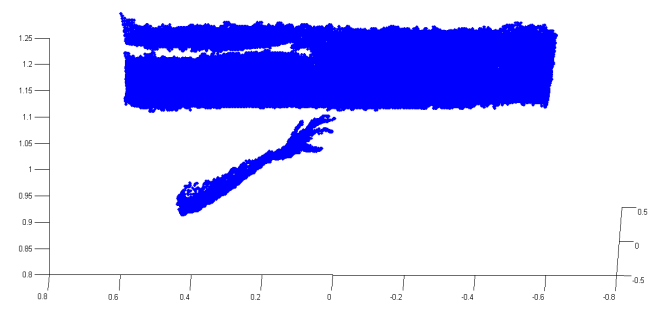

(a)

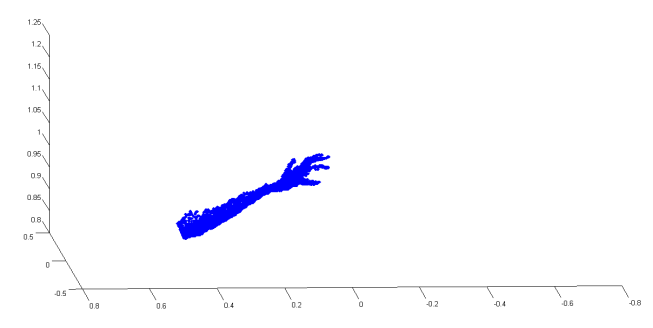

(c)

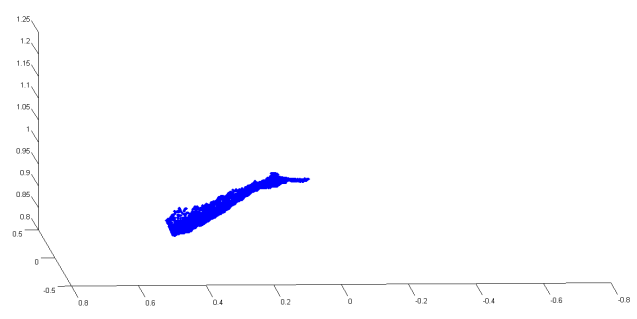

(b)

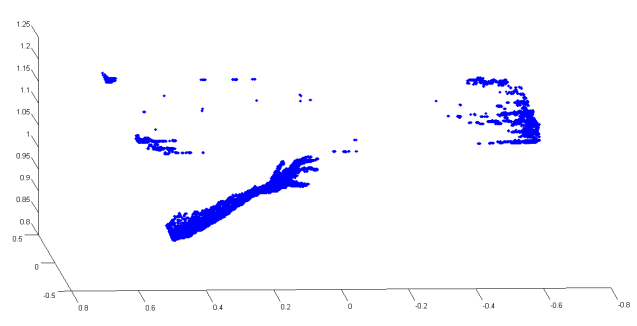

(d)

Figure 3: Results of applying RANSAC plane fitting algorithm with different threshold values: (a) Original Point Cloud; b) $\tau=0.16$; c) $\tau=0.10$; d) $\tau=0.01$.

DBSCAN. K-means is a popular clustering algorithm that partitions data in a fixed a priori number of clusters. Two steps are iterated recursively until convergence is reached: find $k$ centroids as barycenters of the clusters and associate each point to the nearest centroid. The Euclidean Cluster Extraction [25] is a simple algorithm for clustering a 3-D point cloud based on the Euclidean distance proposed by Rusu. For each point of the data set the algorithm, basically, searches for the nearest points inside a sphere of a fixed radius $r$. The number of clusters is found by processing all the points of the data set whereby only the radius of the sphere is required as an input parameter.

DBSCAN (Density Based Spatial Clustering of Application with Noise) [26] is another technique based on density estimation for arbitrary shaped clusters in spatial databases. The algorithm is based on two notions: cluster and noise. DBSCAN starts with an arbitrary starting point in the dataset that has not been visited. If its eps-neighborhood contains a sufficient number of points, it is classified as a core point, otherwise it is labeled as noise. The search continues for all the points in the dataset. DBSCAN is able to differentiate border points from noisy points of a cluster. In fact, if the minimum number of points is recognized later, points previously marked as noise are renamed. The algorithm requires two parameters as input: eps, the radius of the sphere, and minPts, the minimum number of points to mark a cluster as a core object.

In both the latter two algorithms, the nearest neighbor searches have been performed creating a kd-tree for each point cloud. We have used the structure written by Tagliasacchi [27] obtaining a substantial performance improvement for search operations in the point clouds. Fig. 4 shows the results of the segmentation step on a point cloud during a grasping task. 


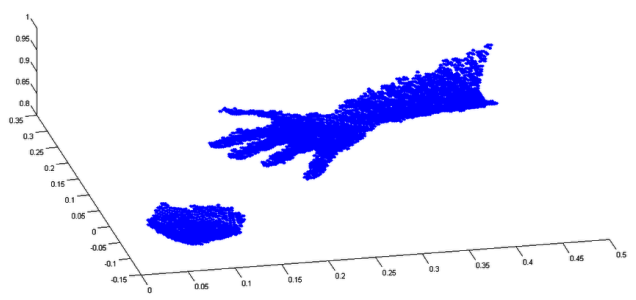

(a)

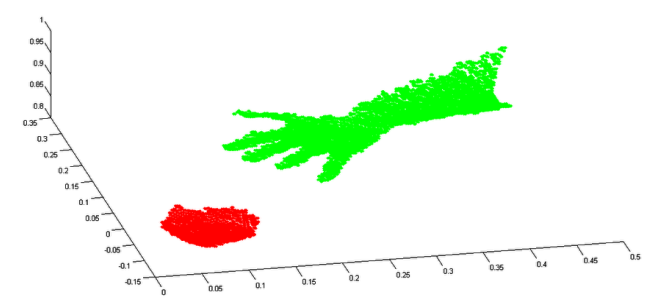

(c)

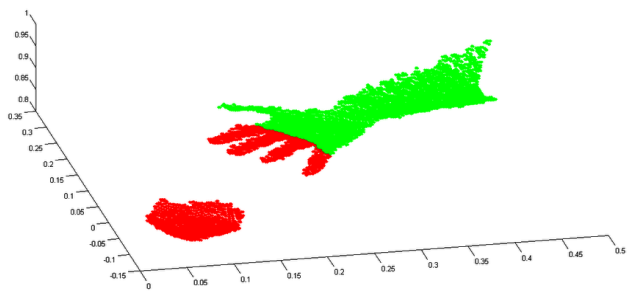

(b)

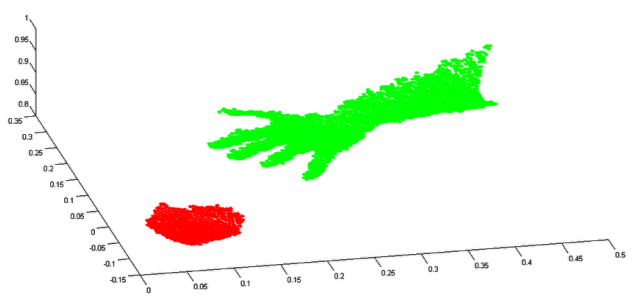

(d)

Figure 4: a) Original Point Cloud; Output of the segmentation step by: b) K-means; c) Euclidean Cluster Extraction; d) DBSCAN.

More generally, if two clusters are close enough, K-means it is not able to divide the objects correctly, whereas the DBSCAN and the Euclidean Cluster Extraction algorithms perform much better. In the following steps we have used the results of the DBSCAN algorithm that in our experiments has appeared to show the best performance.

\subsection{Synthetic hand models generation}

The point clouds prototypes are obtained from the BlenSor software [28], which allows the creation of a point cloud from a $3-\mathrm{D}$ model. Blensor is a simulation software based on Blender that simulates the output of various types of sensors such as LIDAR, Time-of-flight (ToF) and Kinect cameras. A 3-D hand model with 26 DoFs has been built by assembling geometric primitives such as cylinders, spheres and ellipsoids. Each of the four fingers has 4 DoFs while the thumb has 7 DoFs as a result of a different structure. The fingers together have 23 DoFs. The remaining 3 DoFs are from the rotational motion of the palm. The shape parameters of each object are set by taking measurements from a real hand. In order to manipulate such model, a skeleton based on armature objects is created with rigging process. Rigging is the process of attaching a skeleton to a mesh object that allows to deform and to pose it in different ways. Moreover, to remain within feasible movements, hand and finger motion uses static constraints that typically limit the motion of each finger [29], see Fig. 5. The constraints, used to obtain the point cloud of the relative pose, are 


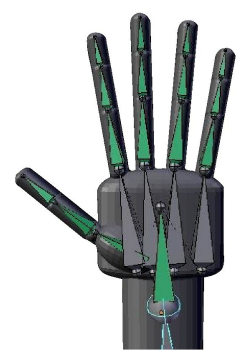

(a)

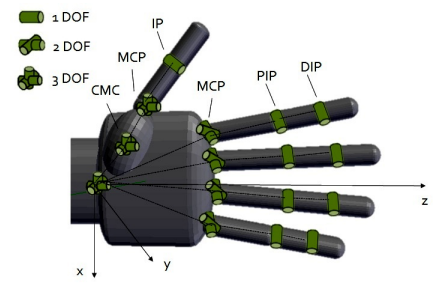

(b)

Figure 5: a) 3-D hand model and corresponding skeleton that controls its deformations (constrained bones are shown in green); b) DOFs of the hand joints.

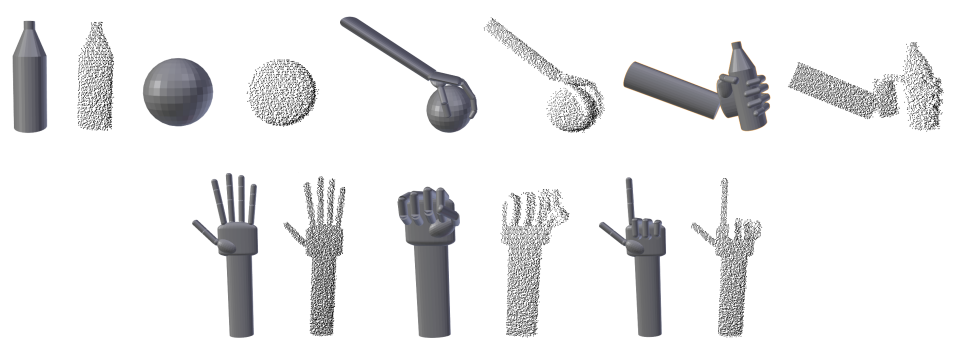

Figure 6: The 3-D models and their corresponding point clouds generated for our dataset.

the following:

$$
\begin{array}{r}
0^{\circ} \leq \theta_{M C P-F} \leq 90^{\circ}, \\
0^{\circ} \leq \theta_{P I P} \leq 110^{\circ}, \\
0^{\circ} \leq \theta_{D I P} \leq 90^{\circ}, \\
-15^{\circ} \leq \theta_{M C P-A A} \leq 15^{\circ} .
\end{array}
$$

In Fig. 6 some of the 3-D models are shown with their associated point clouds that have been used in building the prototypes for our dataset. In particular, we have considered three typical gestures of the hand (palm, fist and gun) and two "interaction" models when the hand interacts with an object.

\subsection{Matching}

The next step consists of finding the optimal registration between the point clouds in the dataset and the point cloud obtained by the clustering algorithm. To perform the matching between the models and the data acquired by the Kinect sensor, the ICP algorithm [30] has been used. In this regard, we provide a detailed description of our system in two typical cases: the hand performs simple gestures and the hand interacts with an object. Due to many problems, such as local minima, noise and partial overlap, the ICP algorithm may converge slowly or not converge at all. In addition, the position of the hand in the space is not known at the beginning of the simulation. For these reasons, we propose a modification 
of the ICP algorithm considering its "stochastic" version.

The goal of the ICP algorithm is to bring two point clouds as close as possible to each other. The algorithm at each iteration step, selects the nearest points of a cloud (the model, in our case) respect to the other one (the input data) and calculates the transformation, represented by a rotation matrix $\mathbf{R}$ and a translation vector $\mathbf{t}$, that minimize the following equation:

$$
E(\mathbf{R}, \mathbf{t})=\sum_{i=1}^{N_{p}} \sum_{j=1}^{N_{m}} w_{i, j}\left\|\mathbf{p}_{i}-\left(\mathbf{R m}_{j}+\mathbf{t}\right)\right\|^{2},
$$

where $\mathbf{M}=\left\{m_{1}, \ldots, m_{N_{m}}\right\}$ is the model's point cloud, $\mathbf{P}=\left\{p_{1}, \ldots, p_{N_{p}}\right\}$ is the input point cloud after the segmentation step and $w_{i, j} \in\{0,1\}$ represents the point correspondences. This equation represents the objective function that is minimized at each iteration; in particular, it represents the sums of squared distance of the model points to the data points (known as point-to-point minimization). A closed form solution for this rigid body transformation can be calculated using a method based on the singular value decomposition (SVD). The centroids and the deviations from such centroids are given by:

$$
\begin{gathered}
\mu_{m}=\frac{1}{N_{m}} \sum_{i=1}^{N_{m}} \mathbf{m}_{i}, \quad \mu_{p}=\frac{1}{N_{p}} \sum_{i=1}^{N_{p}} \mathbf{p}_{i}, \\
\mathbf{M}^{\prime}=\left\{\mathbf{m}_{i}-\mu_{m}\right\}=\left\{\mathbf{m}_{i}^{\prime}\right\}, \quad \mathbf{P}^{\prime}=\left\{\mathbf{p}_{i}-\mu_{p}\right\}=\left\{\mathbf{p}_{i}^{\prime}\right\} .
\end{gathered}
$$

Considering the SVD of the matrix $\mathbf{N}$,

$$
\mathbf{N}=\sum_{i=1}^{N_{m}} \mathbf{p}_{i}^{\prime} \mathbf{m}_{i}^{\prime T}=U \Sigma V^{T}
$$

where $U, V \in \mathbb{R}^{3 x 3}$ are unitary and $\Sigma$ is the matrix of the singular values of $\mathbf{N}$, if $\operatorname{rank}(\mathbf{N})=$ 3 , the optimal solution of $\mathrm{E}(\mathbf{R}, \mathbf{t})$ is unique and given by:

$$
\begin{gathered}
\mathbf{R}=\mathbf{U V}^{T}, \\
\mathbf{t}=\mu_{p}-\mathbf{R} \mu_{m} .
\end{gathered}
$$

The minimal value of the error function is

$$
E(\mathbf{R}, \mathbf{t})=\sum_{i=1}^{N_{m}}\left(\left\|\mathbf{p}_{i}^{\prime}\right\|^{2}+\left\|\mathbf{m}_{i}^{\prime}\right\|^{2}\right)-2\left(\sigma_{1}+\sigma_{2}+\sigma_{3}\right),
$$

where $\sigma_{1} \geq \sigma_{2} \geq \sigma_{3}$ are the singular values of $\mathbf{N}$. Several methods have been proposed to increase the robustness of the ICP algorithm $[31 ; 32 ; 33]$. Our approach consists in adding a normally distributed noise and a random rotation matrix to perturb the position of the model's point clouds in order to obtain a good alignment at the first frame (a similar approach was proposed in [34]). More specifically: $\mathbf{m}_{j}^{\prime}=\mathbf{R}_{\text {rand }} \mathbf{m}_{j}+\mathbf{s}, \quad j=1, \ldots, N_{m}$, where $\mathbf{s}$ has zero mean and diagonal covariance matrix $\sigma^{2} I$ and $\mathbf{R}_{\text {rand }}$ is a random rotation matrix. The parameter $\sigma$ has been set to 0.1 for the experiments and its value has been determined experimentally. We have used the algorithm proposed by Arvo [35] to generate 
a random rotation matrix. The stochastic version of the ICP algorithm operates as reported in Algorithm 1: the model $\mathbf{M}$ is perturbed as discussed before, and then it is applied the standard version of the ICP algorithm on such perturbed model with respect to the input data $\mathbf{P}$. This operation is repeated a fixed number of times. Among all perturbed models, the one closest to the data captured by the sensor is chosen. Finally, the standard ICP algorithm is repeated on such model using as stopping criteria the two parameters max_iter and err. The input parameters $n$ and err have been set respectively to 50 and 1e-5. In Fig. 7 the first four perturbed models obtained by applying the stochastic ICP algorithm are shown. It is important to notice that this procedure can be parallelized because the model is perturbed using different values of $\mathbf{s}$ and $\mathbf{R}_{\text {rand }}$ every time.

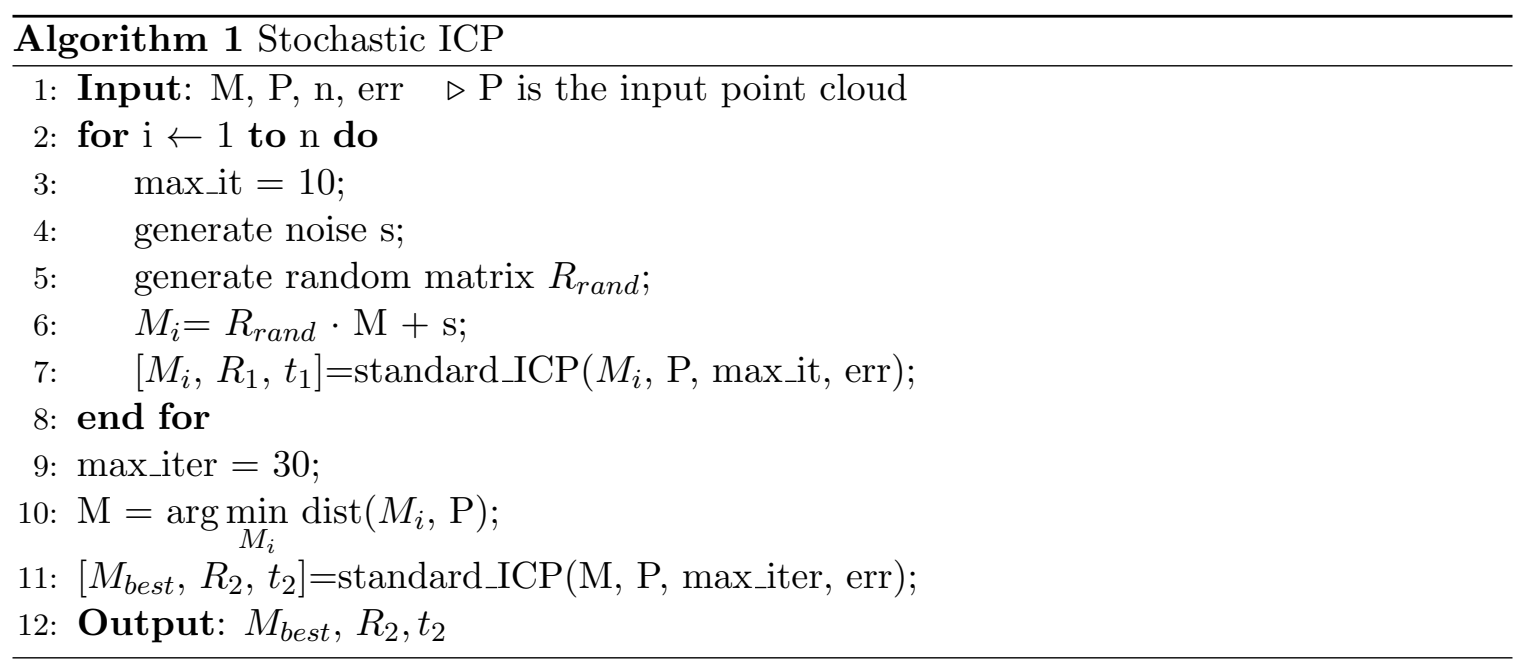

\subsubsection{MATCHING STRATEGY}

Using the stochastic ICP algorithm presented above, two different strategies have been developed. When the hand does not interact with an object we adopt the following strategy (Algorithm 2): in the first frame, the stochastic ICP algorithm is performed, using the three static gestures (palm, gun, fist) in the dataset. Then, from the model that presents the best matching with the acquired data, the translation vector and the rotation matrix are obtained. All models are updated with these two parameters because they have been created using the same viewpoint and the same position. Starting from the next frame, to the end of simulation, the standard ICP algorithm is performed since it converges for relatively small translations and rotations around all axes. In fact, we can reasonably assume that the hand executes small movements between two consecutive frames. All models are still updated with the pose estimated in the previous frame.

When the hand interacts with an object, we assume to begin with two separate clusters. In this case, we need an initial phase of detection. Firstly, the stochastic ICP algorithm is applied to determine which cluster is the object by performing the matching algorithm between the objects' point clouds in the dataset and each of the two clusters. The cluster with the minimum error is labeled as object $\left(C_{1}\right)$; therefore, the other cluster is labeled as hand $\left(C_{2}\right)$. Then, the stochastic ICP algorithm is repeated on the hand cluster $\left(C_{2}\right)$ using 

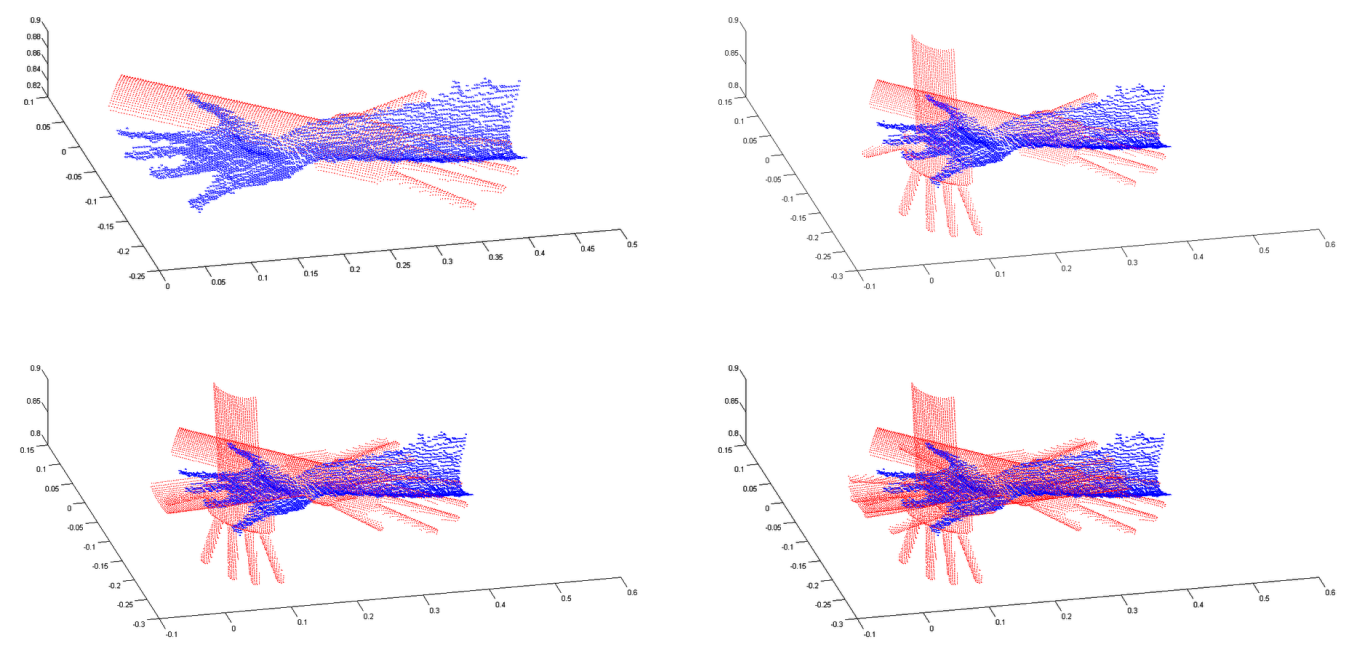

Figure 7: First four perturbed models obtained by applying the "stochastic" version of the ICP algorithm. Among all perturbed models, the closest one to the data captured by the sensor is chosen.

the three static hand gestures in the dataset to determine the hand pose. As long as the two clusters are separated, the standard version of the ICP algorithm is applied, using the three models for the hand cluster and the detected object's model for the other one to update the pose of both models. When the clusters are merged into a single one, the stochastic ICP algorithm is applied using only the two "interaction" models in the dataset. In this case, the stochastic ICP allows to obtain a more robust matching. The strategy is reported in detail in Algorithm 3.

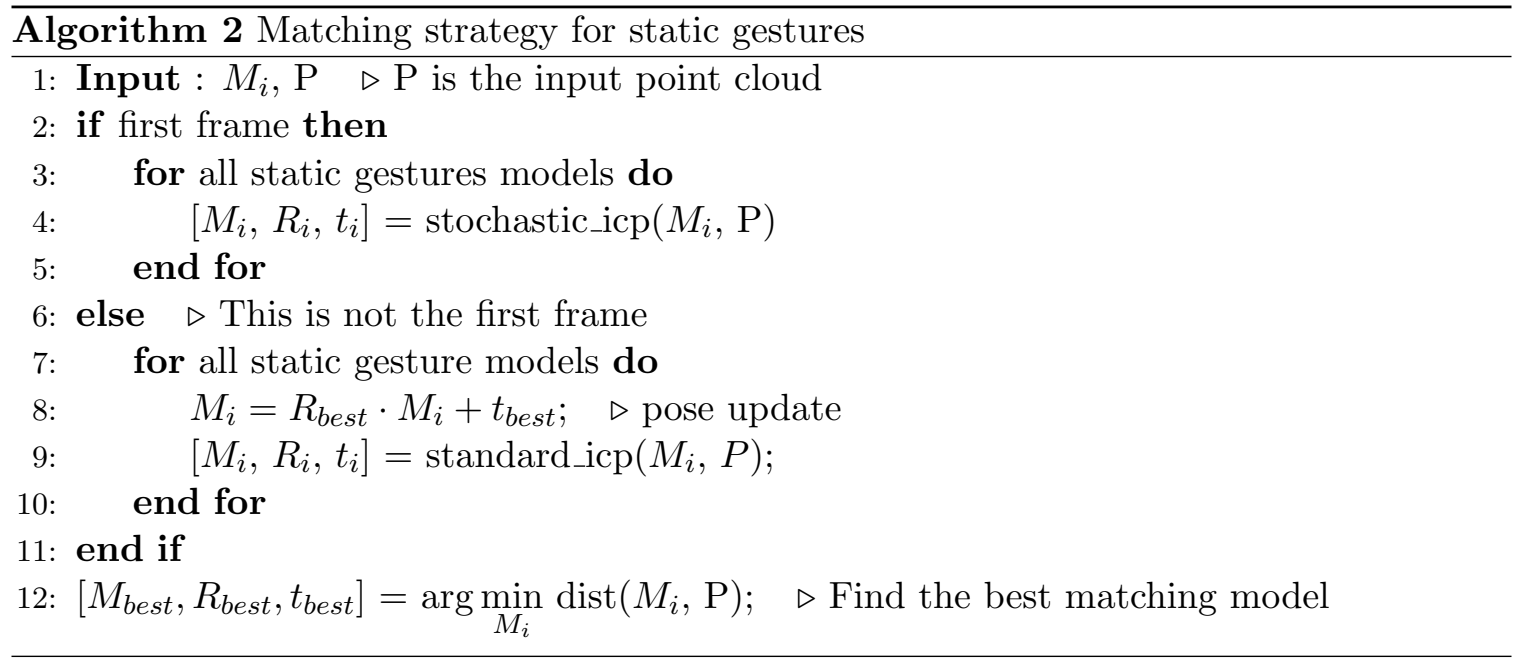




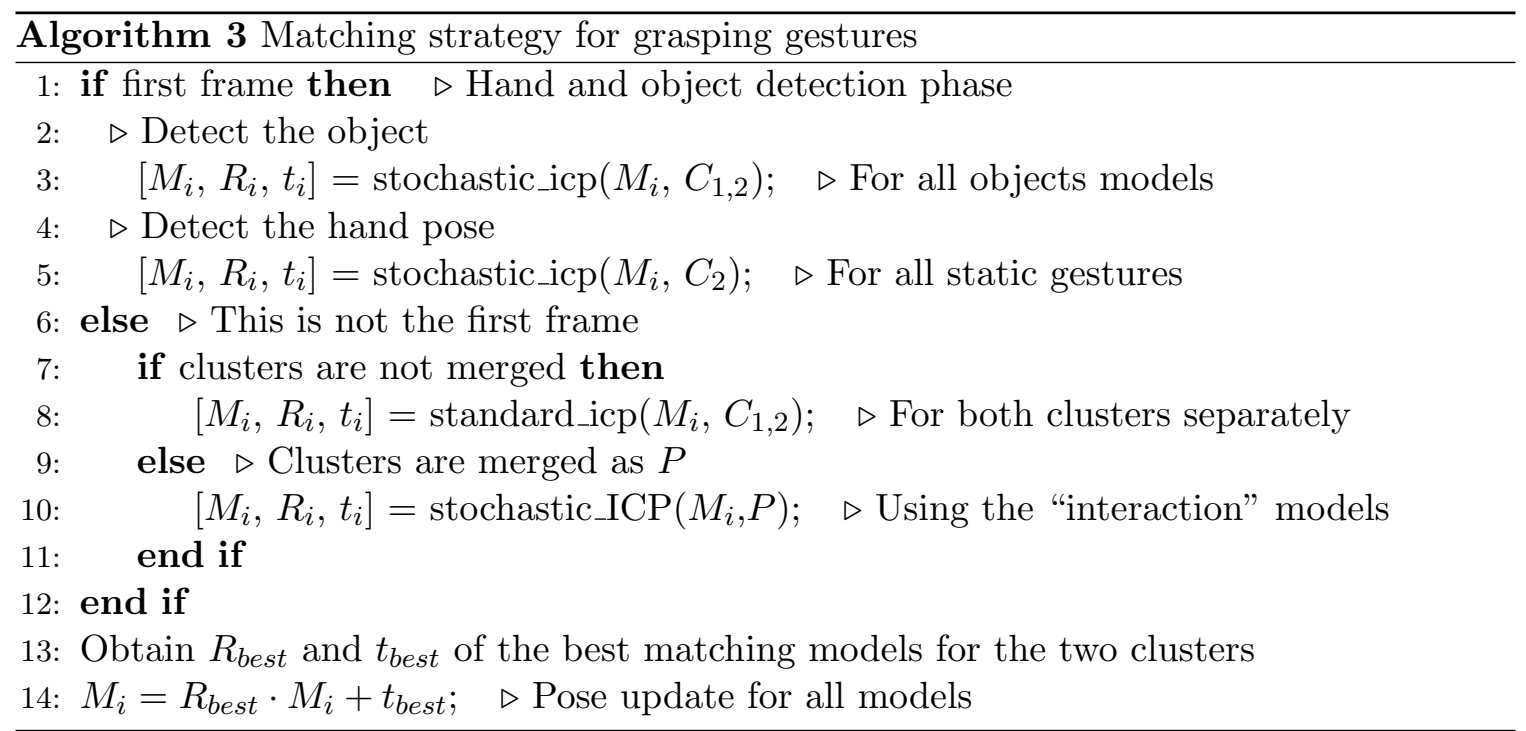

\section{Experimental results}

In order to evaluate the accuracy of the system, we have used the RMS error obtained by considering the Euclidean distance between each point of the selected model and the input data after the clustering step. Since the input data typically contain a number of points greater than the models, because of the presence of the arm or the other body parts, this distance is evaluated considering the nearest points of both point clouds. In addition, a measure of probability $p_{i}[k]$, of the $\mathrm{i}$-th model at the $\mathrm{k}$-th frame, has been defined using the corresponding RMS error as follows:

$$
p_{i}[k]=\frac{\frac{1}{R M S E_{i}[k]}}{\sum_{i=1}^{N_{\text {models }}} \frac{1}{R M S E_{i}[k]}} .
$$

Fig. 8 show the interface of the proposed system. The probabilities are plotted using a bar graph updated every frame.

Even though the system does not require an explicit initialization step, we have used an easily recognizable hand pose represented by a palm completely visible at the beginning of simulation. In fact, the system does not perform a real tracking and we considered only a limited number of poses. In the next two sections, the experimental results of our approach are presented. Fig. 9 shows both the qualitative results and the corresponding RMS errors.

\subsection{Static gestures}

The hand is positioned approximately at a distance of $1.8 \mathrm{~m}$ from the Kinect sensor. In this case, the system is able to recognize the three models in the dataset. In fact, the RMS error is less than $10 \mathrm{~mm}$ and it remains constant during all the simulation since the gestures are distinguishable and correspond exactly to the synthetic models. When the hand moves by rotating, the error fluctuates more even if the correct pose is still recognized. In the last part of simulation, the error is greater and the wrong model is selected mainly because of 


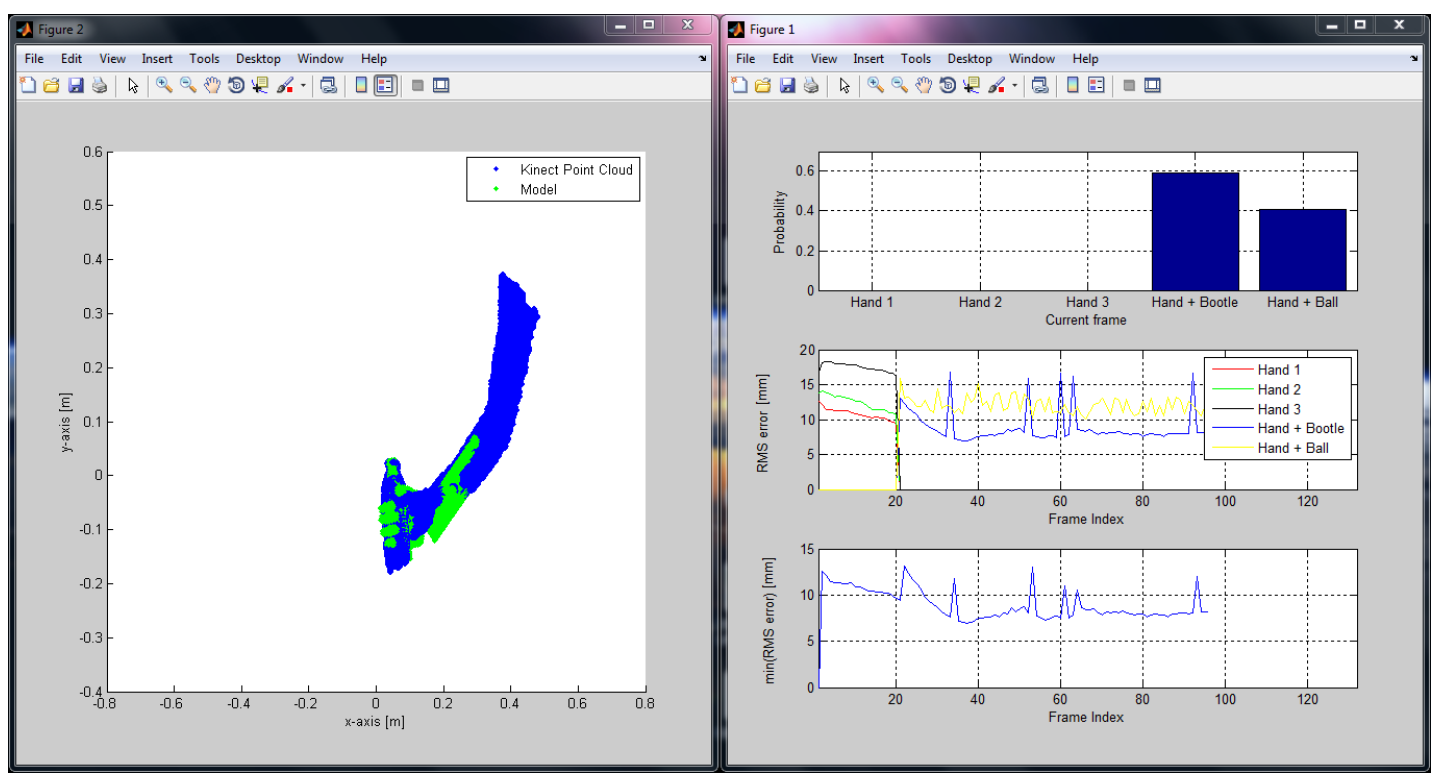

Figure 8: Interface of the proposed hand pose estimation system. Left side: result of the matching step. Right side: probability and RMS error of each model and RMS error of the selected model. The model with the highest probability is plotted (in green) in the same figure of the clustered input data.

a high rotation of the hand that no longer matches the models in the dataset. The visible part, which is captured by the Kinect, is effectively only the profile of the hand and a greater rotation of the model, even if it is correct, results in an increase of the RMS error.

\subsection{Grasping objects}

Finally, we have tested our system when the hand interacts with an object. In particular, we have considered the grasping of objects easily obtainable in $3-\mathrm{D}$ such as a ball and a bottle. For this experiments, we have replaced the three gestures (palm, gun, fist) with three intermediate poses representing the hand that comes close to the objects. The approach works quite well in the initial phase, recognizing the correct pose of the hand and the objects. As soon as the clustering algorithm returns a single cluster in the scene, only the interaction models are considered. As expected, the approach shows obvious problems in recognizing the correct model, when the clusters begin to join, because they are not completely merged. For the grasping ball task, the RMS error remains less than $10 \mathrm{~mm}$, as in the above experiments. However, for some frames, the correct model is chosen but the estimated pose is wrong, primarily because of a lack of a real tracking. For the grasping bottle task some RMS error peaks are visible due to the presence of the entire arm that increases the surface area that needs to be considered for matching. 


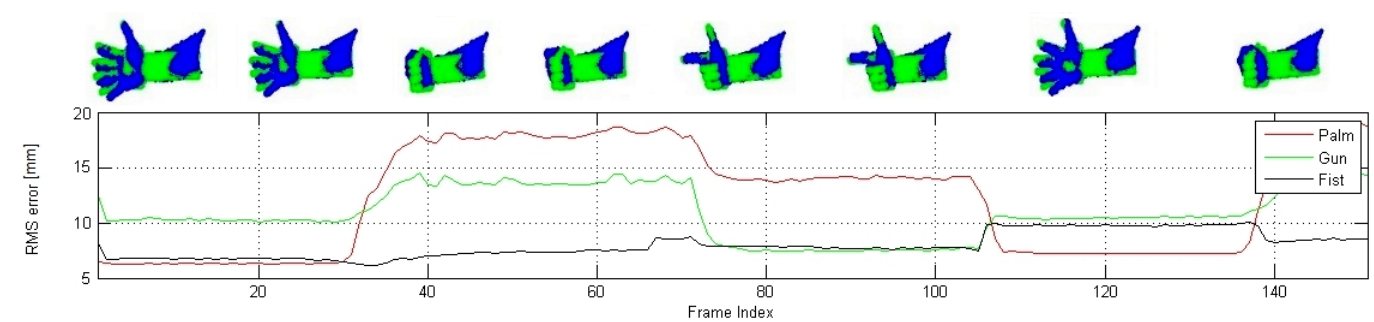

(a)

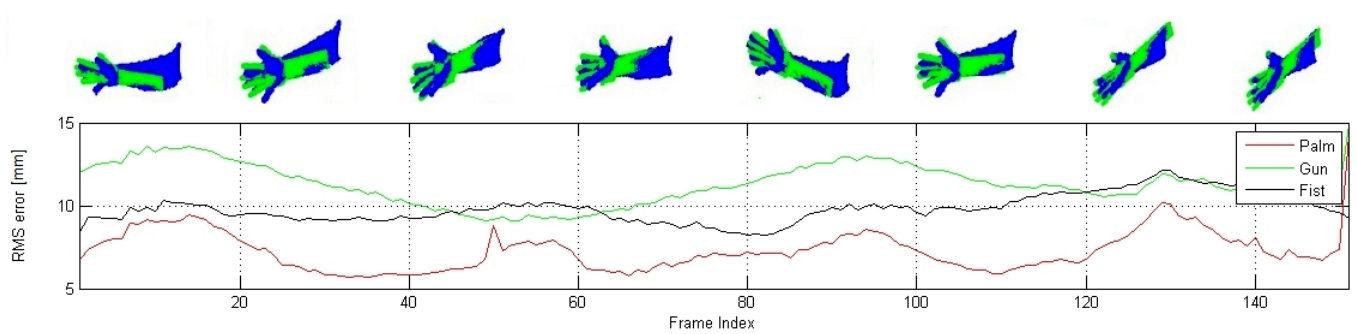

(b)

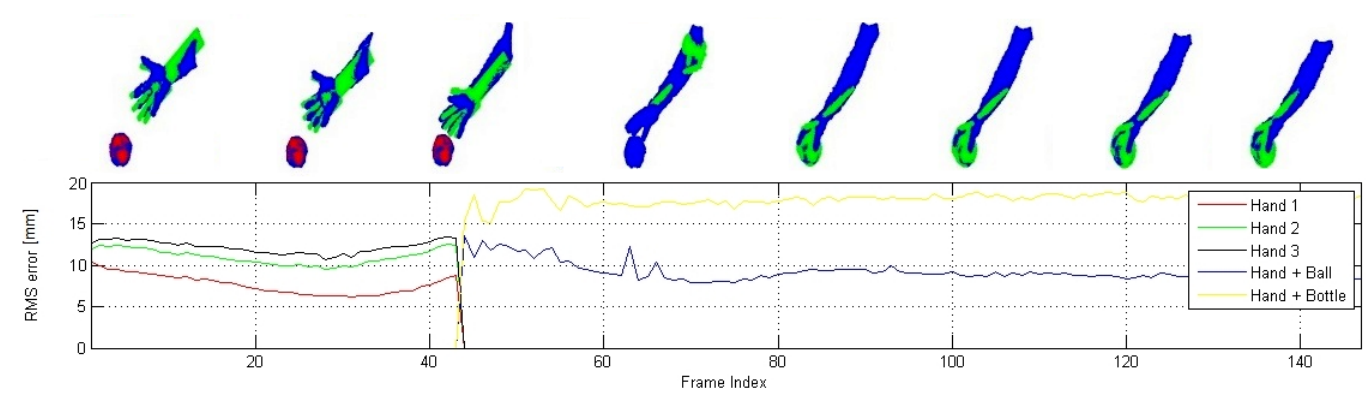

(c)

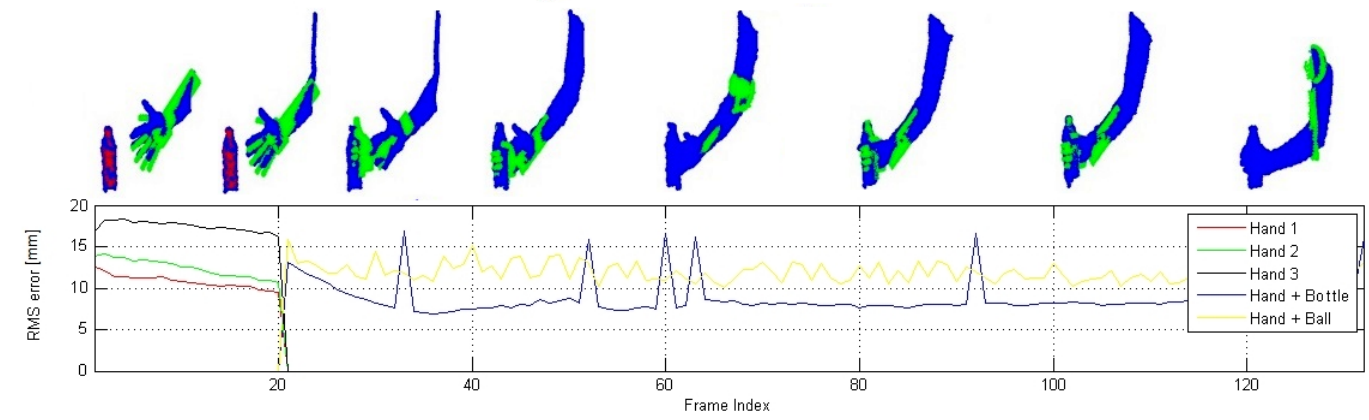

(d)

Figure 9: Sample image sequences of the matching step and the corresponding RMS error:

a) Hand performs static gestures; b) Hand performs rotations showing the palm;

c) Ball grasping task; b) Bottle grasping task. 


\section{Conclusions and future work}

In this work, we have presented a hand pose estimation system which is able to recognize a limited number of hand poses and interaction scenarios combining algorithms widely used in computer vision. The experimental results are very promising and demonstrate the effectiveness of the proposed approach. The system is able to provide a reasonable 3-D hand pose estimation using predefined models, as confirmed by the RMS error, also considering more complex scenarios in which the hand interacts with different objects.

In our future work, possible improvements will be investigated. First of all, the system performance could be improved using parallelized code on GPU or by means of the use of the Point Cloud Library (PCL) [36], an open-source library of algorithms specifically created for point-cloud processing tasks and 3-D geometry processing. Moreover, a larger dataset of typical gestures will be designed. Furthermore, an increase in accuracy could be achieved by implementing a tracking module to support the pose estimation.

\section{References}

[1] Ali Erol, George Bebis, Mircea Nicolescu, Richard D. Boyle, and Xander Twombly. Vision-based hand pose estimation: A review. Computer Vision and Image Understanding, 108(1-2):52 - 73, 2007. ISSN 1077-3142. doi: 10.1016/j.cviu.2006.10.012.

[2] Zhengyou Zhang. Microsoft kinect sensor and its effect. IEEE MultiMedia, 19(2):4-10, April 2012. ISSN 1070-986X. doi: 10.1109/MMUL.2012.24.

[3] Nimble VR. 3Gear Systems interface. http://nimblevr.com/, 2014.

[4] Leap Motion. Leap Motion Controller. https://www.leapmotion.com/, 2014.

[5] Creative. Creative Senz3D. http://en.europe.creative.com/p/web-cameras/ creative-senz3d/, 2014.

[6] B. Stenger, P.R.S. Mendonca, and R. Cipolla. Model-based 3d tracking of an articulated hand. In Computer Vision and Pattern Recognition, 2001. CVPR 2001. Proceedings of the 2001 IEEE Computer Society Conference on, volume 2, pages II-310-II-315 vol.2, 2001. doi: 10.1109/CVPR.2001.990976.

[7] James M. Rehg and Takeo Kanade. Visual tracking of high dof articulated structures: An application to human hand tracking. In Proceedings of the Third European Conference-Volume II on Computer Vision - Volume II, ECCV '94, pages 35-46, London, UK, UK, 1994. Springer-Verlag. ISBN 3-540-57957-5.

[8] M. de La Gorce, D.J. Fleet, and N. Paragios. Model-based 3d hand pose estimation from monocular video. Pattern Analysis and Machine Intelligence, IEEE Transactions on, 33(9):1793-1805, Sept 2011. ISSN 0162-8828. doi: 10.1109/TPAMI.2011.33.

[9] T.E. de Campos and D.W. Murray. Regression-based hand pose estimation from multiple cameras. In Computer Vision and Pattern Recognition, 2006 IEEE Computer Society Conference on, volume 1, pages 782-789, June 2006. doi: 10.1109/CVPR.2006.252. 
[10] V. Athitsos and S. Sclaroff. Estimating 3d hand pose from a cluttered image. In Computer Vision and Pattern Recognition, 2003. Proceedings. 2003 IEEE Computer Society Conference on, volume 2, pages II-432-9 vol.2, June 2003. doi: 10.1109/CVPR. 2003.1211500.

[11] R. Rosales, V. Athitsos, L. Sigal, and S. Sclaroff. 3d hand pose reconstruction using specialized mappings. In Computer Vision, 2001. ICCV 2001. Proceedings. Eighth IEEE International Conference on, volume 1, pages 378-385 vol.1, 2001. doi: 10.1109/ ICCV.2001.937543.

[12] Zhenyao Mo and U. Neumann. Real-time hand pose recognition using low-resolution depth images. In Computer Vision and Pattern Recognition, 2006 IEEE Computer Society Conference on, volume 2, pages 1499-1505, 2006. doi: 10.1109/CVPR.2006.237.

[13] S. Malassiotis and M.G. Strintzis. Real-time hand posture recognition using range data. Image and Vision Computing, 26(7):1027 - 1037, 2008. ISSN 0262-8856. doi: 10.1016/j.imavis.2007.11.007.

[14] Xia Liu and K. Fujimura. Hand gesture recognition using depth data. In Automatic Face and Gesture Recognition, 2004. Proceedings. Sixth IEEE International Conference on, pages 529-534, May 2004. doi: 10.1109/AFGR.2004.1301587.

[15] P. Suryanarayan, A. Subramanian, and D. Mandalapu. Dynamic hand pose recognition using depth data. In Pattern Recognition (ICPR), 2010 20th International Conference on, pages 3105-3108, Aug 2010. doi: 10.1109/ICPR.2010.760.

[16] Nikolaos Kyriazis Iason Oikonomidis and Antonis Argyros. Efficient model-based 3d tracking of hand articulations using kinect. In Proceedings of the British Machine Vision Conference, pages 101.1-101.11. BMVA Press, 2011. ISBN 1-901725-43-X. doi: 10.5244/C.25.101.

[17] Stan Melax, Leonid Keselman, and Sterling Orsten. Dynamics based 3d skeletal hand tracking. In Proceedings of Graphics Interface 2013, GI '13, pages 63-70, Toronto, Ont., Canada, Canada, 2013. Canadian Information Processing Society. ISBN 978-14822-1680-6.

[18] Qiushi Fu and M. Santello. Towards a complete description of grasping kinematics: A framework for quantifying human grasping and manipulation. In Engineering in Medicine and Biology Society,EMBC, 2011 Annual International Conference of the IEEE, pages 8247-8250, Aug 2011. doi: 10.1109/IEMBS.2011.6092033.

[19] H. Hamer, J. Gall, T. Weise, and L. Van Gool. An object-dependent hand pose prior from sparse training data. In Computer Vision and Pattern Recognition (CVPR), 2010 IEEE Conference on, pages 671-678, June 2010. doi: 10.1109/CVPR.2010.5540150.

[20] Takashi Chikamasa. Simulink Support for Kinect. MATLAB Central File Exchange. http://uk . mathworks.com/matlabcentral/fileexchange/32318-simulinksupport-for-kinect/, 2014. 
[21] P. Trivedi, T. Agarwal, and K. Muthunagai. Mc-ransac: A pre-processing model for ransac using monte carlo method implemented on a gpu. In Advances in Computing, Communications and Informatics (ICACCI), 2013 International Conference on, pages 1380-1383, Aug 2013. doi: 10.1109/ICACCI.2013.6637380.

[22] C. Distante and G. Indiveri. Ransac-lel: An optimized version with least entropy like estimators. In Image Processing (ICIP), 2011 18th IEEE International Conference on, pages 1425-1428, Sept 2011. doi: 10.1109/ICIP.2011.6115709.

[23] P. D. Kovesi. MATLAB and Octave functions for computer vision and image processing. Centre for Exploration Targeting, School of Earth and Environment, The University of Western Australia. http://www.csse.uwa.edu.au/ pk/research/ matlabfns/, 2014.

[24] Martin A. Fischler and Robert C. Bolles. Random sample consensus: A paradigm for model fitting with applications to image analysis and automated cartography. Commun. ACM, 24(6):381-395, June 1981. ISSN 0001-0782. doi: 10.1145/358669.358692.

[25] Radu Bogdan Rusu. Semantic 3D Object Maps for Everyday Manipulation in Human Living Environments. PhD thesis, Technische Universität München, 2009.

[26] Martin Ester, Hans peter Kriegel, Jrg S, and Xiaowei Xu. A density-based algorithm for discovering clusters in large spatial databases with noise. pages 226-231. AAAI Press, 1996.

[27] Andrea Tagliasacchi. Kd-tree for matlab. MATLAB Central File Exchange. http://uk . mathworks . com/matlabcentral/fileexchange/21512-kd-tree-formatlab/, 2014.

[28] Michael Gschwandtner, Roland Kwitt, Andreas Uhl, and Wolfgang Pree. Blensor: Blender sensor simulation toolbox. In Proceedings of the 7th International Conference on Advances in Visual Computing - Volume Part II, ISVC'11, pages 199-208, Berlin, Heidelberg, 2011. Springer-Verlag. ISBN 978-3-642-24030-0.

[29] J. Lin, Ying Wu, and T.S. Huang. Modeling the constraints of human hand motion. In Human Motion, 2000. Proceedings. Workshop on, pages 121-126, 2000. doi: 10.1109/ HUMO.2000.897381.

[30] Paul J. Besl and Neil D. McKay. A method for registration of 3-d shapes. IEEE Trans. Pattern Anal. Mach. Intell., 14(2):239-256, February 1992. ISSN 0162-8828. doi: $10.1109 / 34.121791$.

[31] C. Langis, M. Greenspan, and G. Godin. The parallel iterative closest point algorithm. In 3-D Digital Imaging and Modeling, 2001. Proceedings. Third International Conference on, pages 195-202, 2001. doi: 10.1109/IM.2001.924434.

[32] Ce Li, Jianru Xue, Shaoyi Du, and Nanning Zheng. A fast multi-resolution iterative closest point algorithm. In Pattern Recognition (CCPR), 2010 Chinese Conference on, pages 1-5, Oct 2010. doi: 10.1109/CCPR.2010.5659287. 
[33] Ce Li, Jianru Xue, Nanning Zheng, Shaoyi Du, Jihua Zhu, and Zhiqiang Tian. Fast and robust isotropic scaling iterative closest point algorithm. In Image Processing (ICIP), 2011 18th IEEE International Conference on, pages 1485-1488, Sept 2011. doi: 10.1109/ICIP.2011.6115724.

[34] G.P. Penney, P.J. Edwards, A.P. King, J.M. Blackall, P.G. Batchelor, and D.J. Hawkes. A stochastic iterative closest point algorithm (stochasticp). In WiroJ. Niessen and MaxA. Viergever, editors, Medical Image Computing and ComputerAssisted Intervention MICCAI 2001, volume 2208 of Lecture Notes in Computer Science, pages 762-769. Springer Berlin Heidelberg, 2001. ISBN 978-3-540-42697-4. doi: 10.1007/3-540-45468-3_91.

[35] James Arvo. Fast random rotation matrices. In David Kirk, editor, Graphics Gems III, pages 117-120. Academic Press, 1992.

[36] R.B. Rusu and S. Cousins. 3d is here: Point cloud library (pcl). In Robotics and Automation (ICRA), 2011 IEEE International Conference on, pages 1-4, May 2011. doi: 10.1109/ICRA.2011.5980567. 